\title{
Economic Evaluations of Immune Checkpoint Inhibitors for Patients with Non-Small Cell Lung Cancer: A Systematic Review
}

This article was published in the following Dove Press journal: Cancer Management and Research

\author{
$\mathrm{Na} \mathrm{Li} i^{1,2, *}$ \\ Huanrui Zheng ${ }^{1,2, *}$ \\ Bin Zheng ${ }^{1,2}$ \\ Chaoxin Chen iD ${ }^{1,2}$ \\ Hongfu Cai ${ }^{1,2}$ \\ Maobai Liu ${ }^{1,2}$ \\ 'Department of Pharmacy, Fujian Medical \\ University Union Hospital, Fuzhou, Fujian \\ Province, People's Republic of China; \\ ${ }^{2}$ School of Pharmacy, Fujian Medical \\ University, Fuzhou, Fujian Province, \\ People's Republic of China
}

*These authors contributed equally to this work
Correspondence: Maobai Liu Department of Pharmacy, Fujian Medical University Union Hospital, Fuzhou, Fujian Province, People's Republic of China Email liumaobai@I63.com
Objective: This review aimed to assess the quality of available evidence on the economic evaluations of immune checkpoint inhibitors in patients with non-small cell lung cancer (NSCLC) and provide evidence to improve the efficiency of healthcare resources.

Materials and Methods: Literature search was performed using some electronic databases (PubMed, Embase and Cochrane Central Register of Controlled Trials). Final search was performed in December 2019. Study characteristics and results were recorded and compared. The quality of the studies was assessed using the Consolidated Health Economic Evaluation Reporting Standards (CHEERS) checklists. We did not elaborate the restrictions on the target population. We included patients with squamous or non-squamous NSCLC and metastatic or advanced cancer.

Results: Of 98 papers considered, 21 were chosen for this review. Most of them are costeffectiveness analysis. Comparative regimens consisted of either immune checkpoint inhibitor monotherapy, immune checkpoint inhibitor plus chemotherapy, or chemotherapy alone. Fourteen, four, and three studies were about pembrolizumab, nivolumab, and atezolizumab, respectively. The methods mostly used in these studies were modeling and sensitivity analysis. All studies used quality-adjusted life year (QALY) and life years (LY) as outcomes. Most studies were conducted in high-income countries. Based on the willingness to pay threshold, atezolizumab, and pembrolizumab were found to be cost-effective in one and 10 studies, respectively. None of the studies concluded that nivolumab was cost-effective. For quality assessment, all studies fulfilled more than $50 \%$ of the CHEERS checklist.

Conclusion: The included studies indicated that pembrolizumab regimens are cost-effective as first-line treatment for patients with NSCLC in developed countries. Nivolumab and atezolizumab are likely to be cost-effective as second-line treatment but not as first-line treatment.

Keywords: non-small cell lung cancer, economic analyses, immune checkpoint inhibitors, systematic review, PD-L1 positive

\section{Introduction}

Lung cancer is a heterogeneous group of tumors of more than 50 histomorphological subtypes. ${ }^{1}$ Lung cancer remains the most common cancer $(11.6 \%$ of all cancers) and the leading cause of cancer deaths, with over 1.7 million deaths worldwide in $2018 .^{2}$ Over the past few decades, non-small cell lung cancer (NSCLC) and small cell lung cancer (SCLC) are the most frequently used diagnostic terms for lung cancer; NSCLC comprises approximately $80-85 \%$ of all lung cancers. ${ }^{1}$ NSCLC includes squamous cell 
carcinoma, adenocarcinoma, and large cell carcinoma. Cancer cells in non-small cell lung cancer grow and divide more slowly and spread relatively later than those in small cell carcinoma. ${ }^{3}$ Only a small portion of patients with NSCLC is clinically diagnosed at an early stage (stage I or II); over $60 \%$ of patients with lung cancer present with locally advanced or metastatic disease (stage III or IV) at the time of diagnosis, at which point surgical resection may not be an option. ${ }^{1}$

The use of advanced molecular profiling to direct targeted therapies has revolutionized the treatment of NSCLC. Although the treatment for this disease has improved with the development of targeted drugs for patients with amenable mutations, only a small proportion of patients has these mutations, and acquired resistance to targeted therapies inevitably occurs. ${ }^{4,5}$ Thus, immunotherapy started to emerge, and targeting the programmed cell death-1(PD-1) pathway is a promising therapeutic option. The emergence of immunotherapy has expanded the treatment options for patients with NSCLC. ${ }^{6}$ The PD-1 pathway binds PD-1 to its ligands[programmed death-ligand 1 (PD-L1)] on tumor cells and suppresses $\mathrm{T}$ cells through a negative feedback loop, leading to evasion of immune responses. ${ }^{7}$ Immunotherapy prevents the combination of PD-1 and PD-L1. ${ }^{8}$

Although immunotherapy is effective for NSCLC, immunotherapeutic drugs are very expensive. The costeffectiveness of using these drugs for NSCLC remains unclear due to the high cost of them. Immune checkpoint inhibitors for NSCLC include pembrolizumab, nivolumab, atezolizumab, and durvalumab. Several economic evaluations have investigated the costs and consequences of these drugs and reached different conclusions. The costeffectiveness of these drugs are one of the research hotspots in different countries. In October 2016, the US Food and Drug Administration (FDA) approved pembrolizumab for first-line treatment of metastatic NSCLC with a PD-L1 tumor proportion score (TPS) $\geq 50 \%$ (determined by an FDA-approved test) and without epidermal growth factor receptor (EGFR) or anaplastic lymphoma kinase (ALK) genomic tumor aberration. ${ }^{9}$ In April 2019, the FDA expanded the pembrolizumab monotherapy indication for first-line treatment of patients with locally advanced or metastatic NSCLC with a PD-L1 TPS $\geq 1 \%$ based on the data from KEYNOTE-042. ${ }^{10}$ However, cost-effectiveness differs among patients with various PD-L1 expression levels. With the improvement of economic level and clinical studies, the research focus and conclusions have also changed. Hence, the present work aimed to systematic review and assess the quality of the cost-effectiveness data of immune checkpoint inhibitors for NSCLC.

\section{Materials and Methods}

A protocol of our systematic review was published in the International Prospective Register of Systematic Reviews (http://www.crd.york.ac.uk/prospero/; registration number is CRD42020151578).

\section{Publication Search}

The inclusion criteria were as follows: 1) full economic evaluations (cost-minimization, cost-benefit, costeffectiveness, and cost-utility analyses) of immune checkpoint inhibitors for patients with NSCLC, 2) immunotherapies compared with chemotherapy or with each other and those used as first- or second-line treatment, 3) the incremental analysis method was used to decide whether the higher cost of the therapeutic regimen relative to the lower plan can bring a satisfactory incremental income, and 4) use of English language in reporting. We excluded articles that focused on diagnostics and adherence, partial economic evaluations, and conference abstract. Two independent researchers (Zheng and Li) carefully searched PubMed, Embase, and Cochrane Central Register of Controlled Trials (CENTRAL) databases to find economic evaluations of NSCLC that met the inclusion criteria. We searched the databases by using the following search terms: "non-small cell lung cancer," "cost," "immunotherapy," "immune checkpoint inhibitors," "pembrolizumab," "nivolumab," "atezolizumab," and "durvalumab." These terms were embellished slightly to meet the subject heading structure of each database.

\section{Quality Assessment and Data Extraction}

The two researchers (Zheng and Li) independently performed all quality assessments and data extractions. Any disagreement between the two reviewers was discussed with a third author (Chen). The Consolidated Health Economic Evaluation Reporting Standards (CHEERS) checklist $^{11}$ was used to assess the study quality. The 24term checklist was created by the International Society for Pharmacoeconomics and Outcomes Research (ISPOR) and includes the assessment of the following: title and abstract, introduction, methods, results, discussion, and others. According to the CHEERS statement checklist, each "Yes" (fully satisfied), "NS" (partially satisfied), and "No" (not satisfied) are equivalent to $1,0.5$, and 0 points, respectively. 
Scores of 19-24, 13-18, 12-17, and below 12 indicate good, medium, poor, and very poor qualities, respectively. Data were extracted following a predesigned table, which includes study details (author, country, and year of publication), baseline characteristics (target population, intervention, comparator, and outcome measure), methods (time horizon, discount, and model based), and results (type of sensitivity analysis and economic evaluation results).

\section{Results}

\section{Search Results}

Our literature search yielded 98 publications; of which, 68 were retained after excluding duplicates. After we screened the titles and/or abstracts of these publications, 37 were remained. Finally, when we reviewed the full texts, 21 records met our inclusion criteria, 16 of the studies were excluded for the following reasons: not in English language $(n=3)$, not a full report $(n=5)$, review $(n=5)$, and conference abstract $(n=3)$. The flow chart of the literature search is presented in Figure 1.

\section{General Characteristics}

The included studies were published between 2016 and 2019

The majority of the studies were conducted in developed countries (USA, UK, France, Canada, Australia, and
Switzerland). Four studies were conducted in China, and one of them was conducted in the Hong Kong region. Fourteen, ${ }^{21,22,24-31,34,38-40}$ four, ${ }^{33,35-37}$ and three ${ }^{12,23,32}$ studies focused on pembrolizumab, nivolumab, and atezolizumab, respectively. No study investigated durvalumab. Almost all the included studies employed cost-effectiveness analysis. Most of them reported the results in terms of cost per quality-adjusted life years (QALYs) and cost per life year (LY) gained. Among these studies, $16^{12,21-31,34,38-40}$ and $5^{32,33,35-37}$ were about first- and second-line treatments for NSCLC, respectively. One study ${ }^{12}$ had two base cases. We did not make clear restrictions for the target population. We included patients with squamous NSCLC or non-squamous NSCLC and metastatic or advanced cancer. The expression of PD-L1 affects the selection of immune checkpoint inhibitors. Fourteen studies ${ }^{21,22,24-31,34,38-40}$ mentioned positive PD-L1 or high level of PD-L1, whereas one studies ${ }^{36}$ explored the cost-effectiveness of PD-L1 testing. The other studies were not clearly stated. These studies were mainly based on the following clinical trials: KEYNOTE 024, ${ }^{13}$ KEYNOTE 042, ${ }^{10}$ KEYNOTE $189,{ }^{14}$ KEYNOTE 407, ${ }^{15}$ KEYNOTE $010^{5}$, CheckMate $057,{ }^{16}$ CheckMate $017,{ }^{17}$ CheckMate $078,{ }^{18}$ the OAK trial, ${ }^{19}$ and IMpower $150 .^{20}$ The basic characteristics and results of the studies are shown in Tables 1 and 2, respectively.

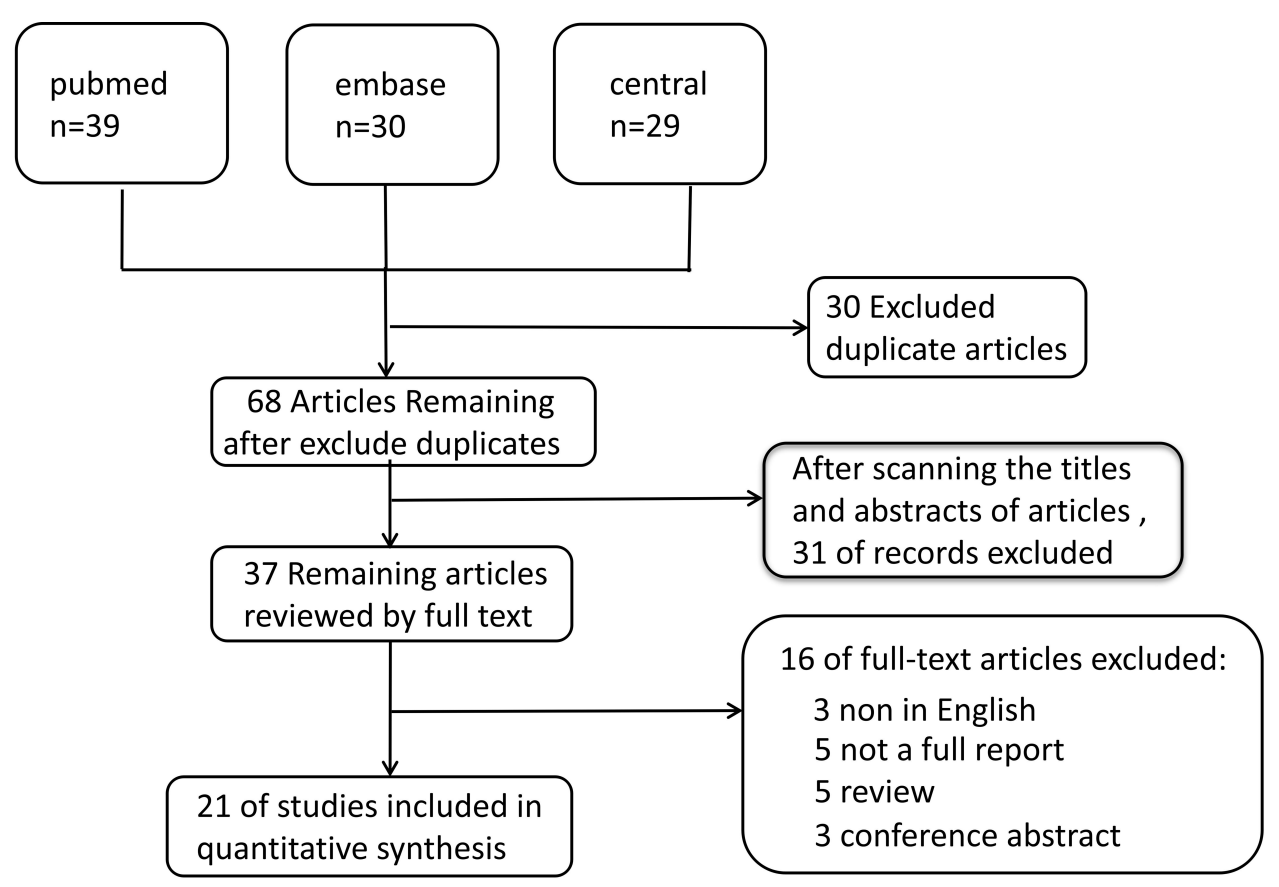

Figure I Flow chart of the systematic literature search.

Notes: Our literature search yielded 98 publications, of which, 68 were retained after excluding duplicates. After we screened the titles and/or abstracts of these publications, 37 were remained. Finally, when we reviewed the full texts, 21 records met our inclusion criteria. 


\begin{tabular}{|c|c|c|c|c|c|c|}
\hline 然 & 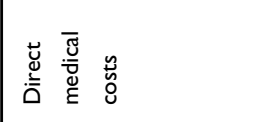 & 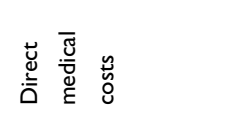 & 总突 & 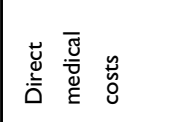 & 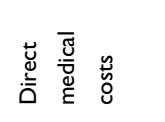 & 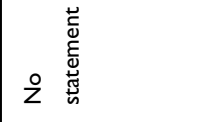 \\
\hline 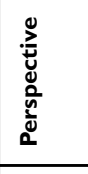 & 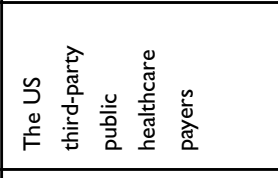 & 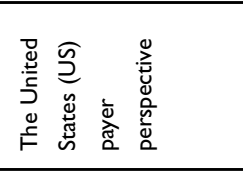 & 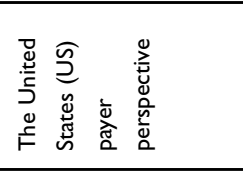 & 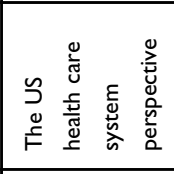 & 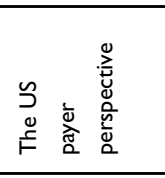 & 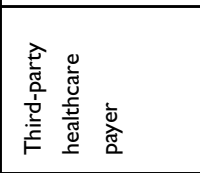 \\
\hline 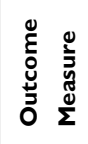 & 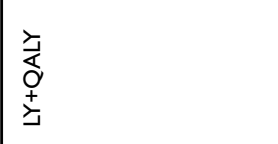 & 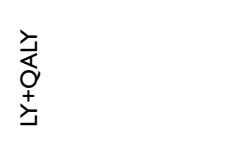 & 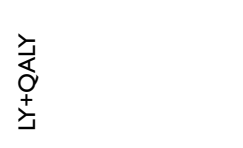 & 忞 & 旁 & 离 \\
\hline 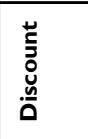 & $\stackrel{\circ}{\circ}$ & $\stackrel{\circ}{\circ}$ & $\stackrel{\circ}{\circ}$ & $\stackrel{\circ}{\circ}$ & 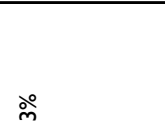 & ஓे \\
\hline 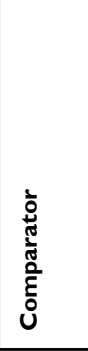 & 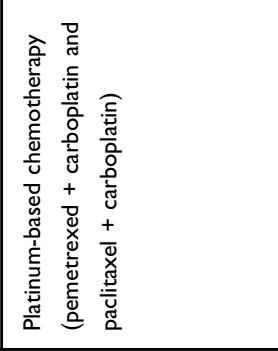 & 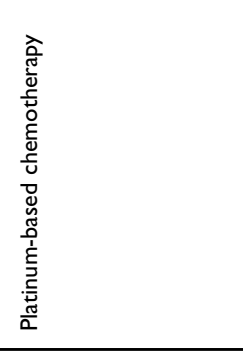 & 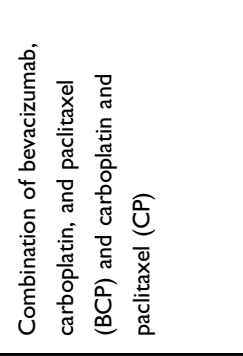 & 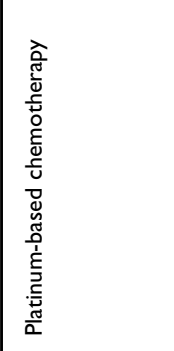 & 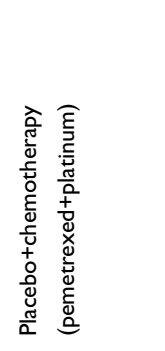 & 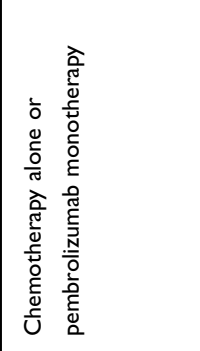 \\
\hline 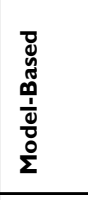 & 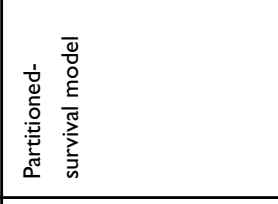 & 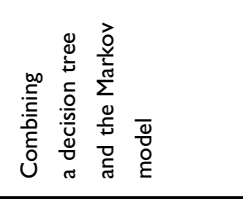 & 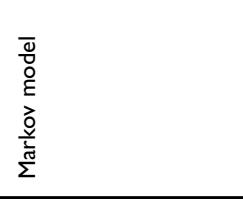 & 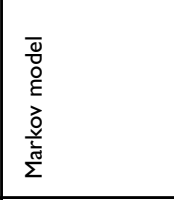 & 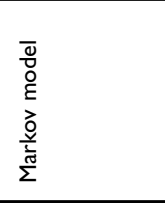 & 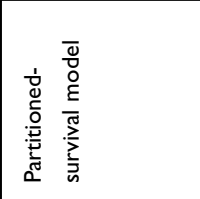 \\
\hline 竞 & 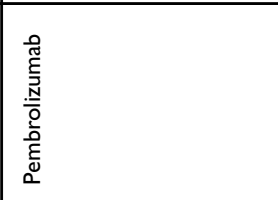 & 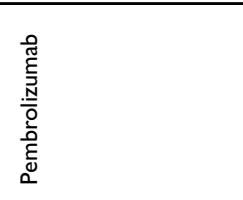 & 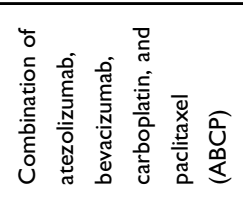 & 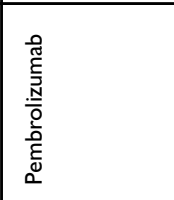 & 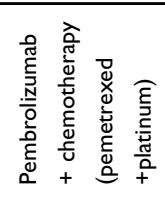 & 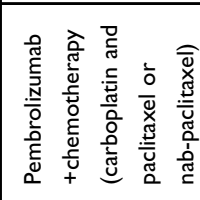 \\
\hline 竞 & 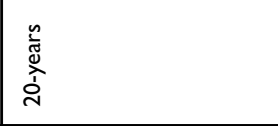 & $\begin{array}{l}\text { 高 } \\
\stackrel{\text { }}{\hat{~}}\end{array}$ & 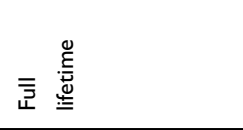 & $\overline{\overline{\bar{i}}}$ & 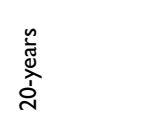 & 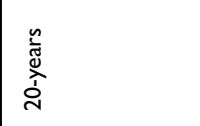 \\
\hline 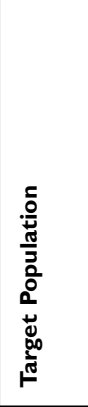 & 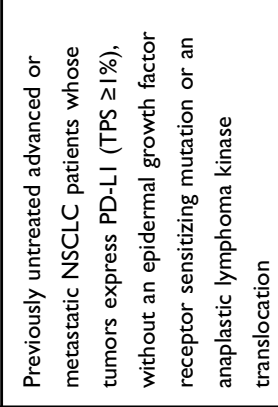 & 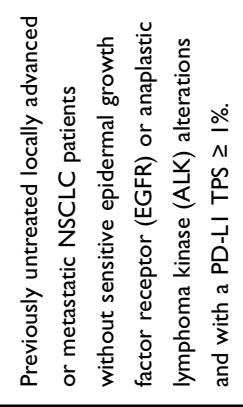 & 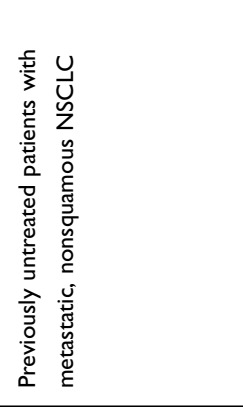 & 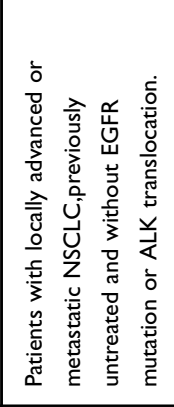 & 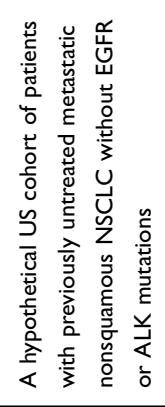 & 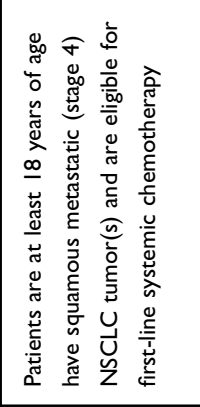 \\
\hline نे & $\stackrel{\leftrightarrow}{د}$ & $\stackrel{\leftrightarrow}{د}$ & $\stackrel{\Delta}{j}$ & $\stackrel{u}{J}$ & $\stackrel{\Delta s}{s}$ & $\stackrel{u}{د}$ \\
\hline ક્ષ & $\stackrel{\circ}{\bar{i}}$ & $\stackrel{\circ}{\bar{\alpha}}$ & $\stackrel{\circ}{\bar{\nu}}$ & $\stackrel{\circ}{\bar{\alpha}}$ & సे & 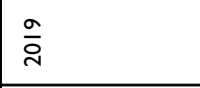 \\
\hline 高 & \begin{tabular}{|l}
$\overline{\bar{c}}_{\mathrm{bo}}$ \\
$\overline{\underline{\underline{I}}}$ \\
$\overline{\underline{I}}$
\end{tabular} & $\overbrace{0}$ & $\begin{array}{l}\overbrace{0} \\
3_{3}^{5}\end{array}$ & 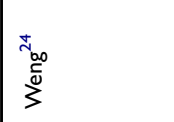 & 紫 & 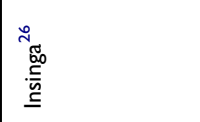 \\
\hline
\end{tabular}




\begin{tabular}{|c|c|c|c|c|c|}
\hline 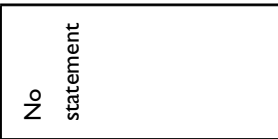 & 点 & 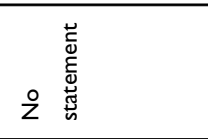 & 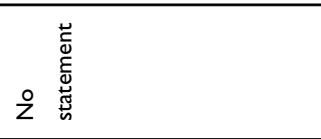 & 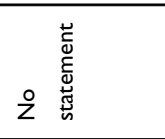 & 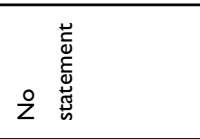 \\
\hline 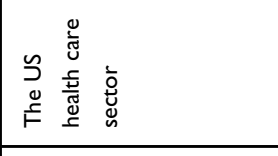 & 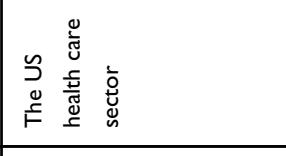 & 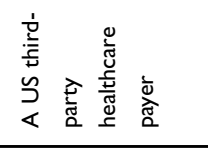 & 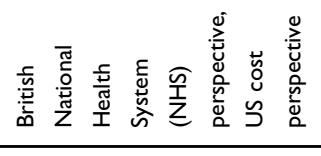 & 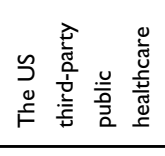 & 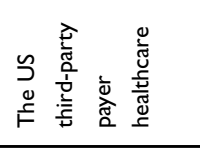 \\
\hline ذ্ & 忞 & $\frac{\grave{d}}{\partial}$ & 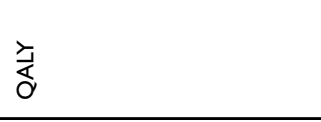 & 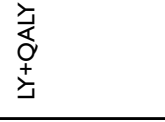 & 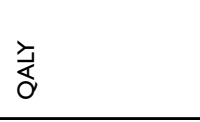 \\
\hline ஓ & $\stackrel{\circ}{\circ}$ & $\stackrel{\circ}{m}$ & & $\stackrel{\circ}{\circ}$ & $\stackrel{\circ}{\circ}$ \\
\hline 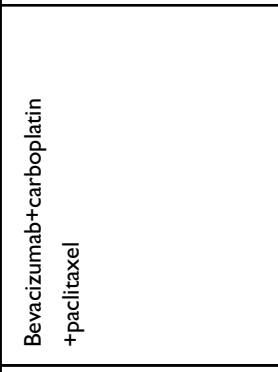 & 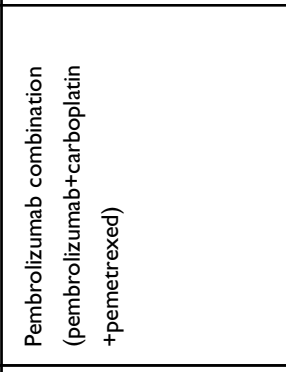 & 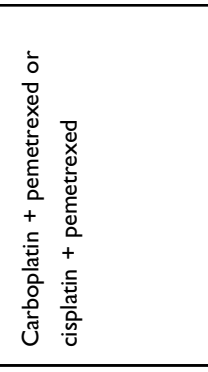 & 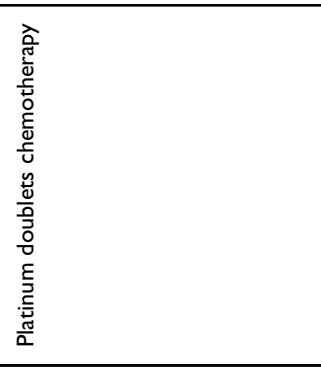 & 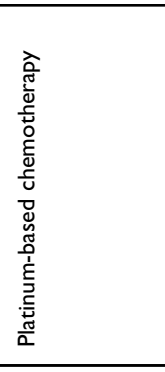 & 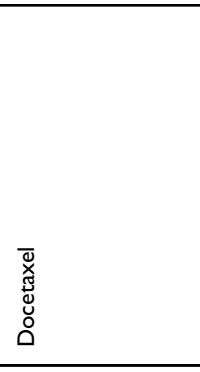 \\
\hline 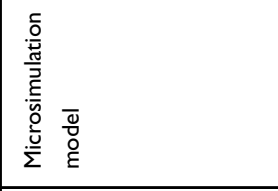 & 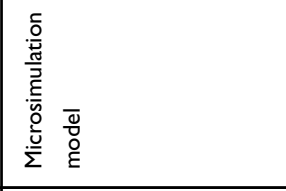 & 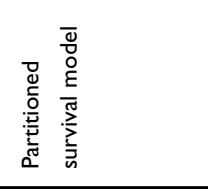 & 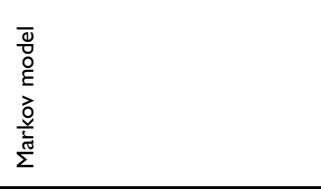 & 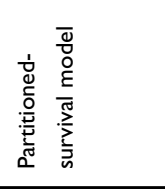 & 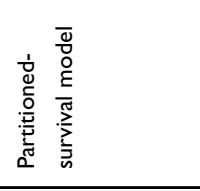 \\
\hline 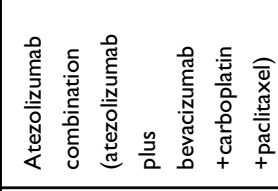 & 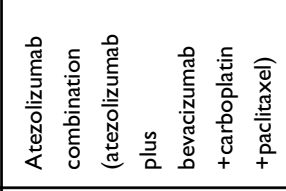 & 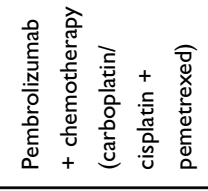 & 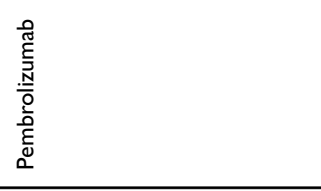 & 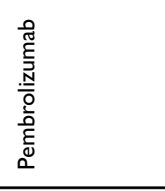 & 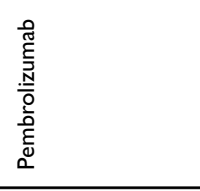 \\
\hline 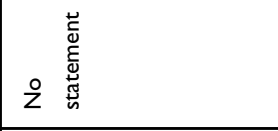 & 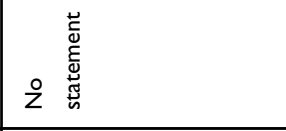 & 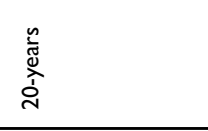 & 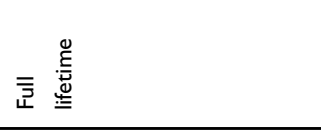 & $\begin{array}{l}\text { 品 } \\
\text { 今े } \\
\text { in }\end{array}$ & 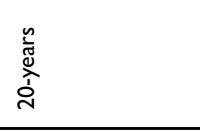 \\
\hline 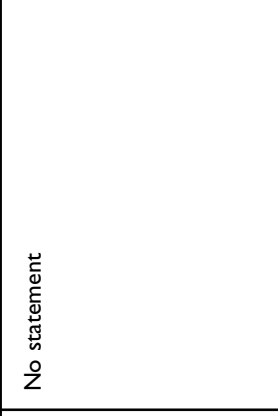 & 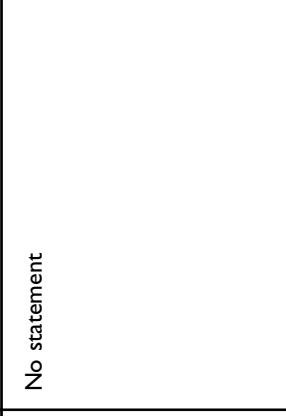 & 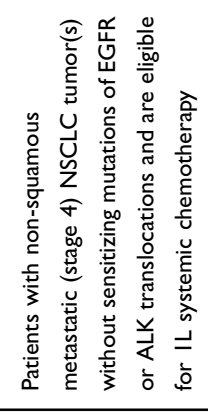 & 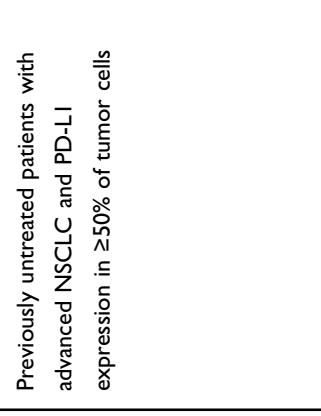 & 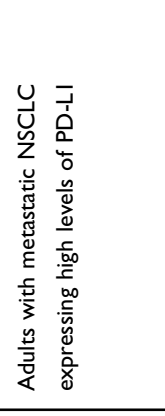 & 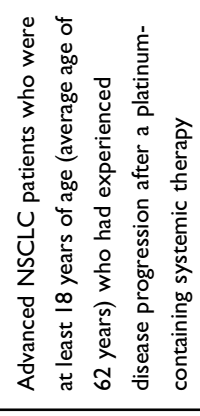 \\
\hline$\stackrel{\dot{j}}{j}$ & $\stackrel{\Delta}{د}$ & $\stackrel{\iota}{د}$ & 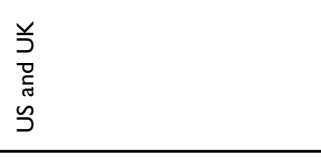 & $\stackrel{\iota}{د}$ & $\stackrel{\dot{s}}{\dot{s}}$ \\
\hline$\stackrel{\circ}{\bar{a}}$ & $\stackrel{\circ}{\bar{\alpha}}$ & $\stackrel{\infty}{\stackrel{2}{2}}$ & $\stackrel{\infty}{\stackrel{2}{c}}$ & $\overline{\bar{a}}$ & $\stackrel{\circ}{\circ}$ \\
\hline$\frac{2}{\bar{d}}$ & $\begin{array}{l}\tilde{z} \\
\frac{\tilde{g}}{\tilde{z}}\end{array}$ & 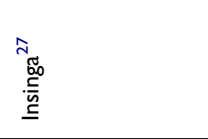 & 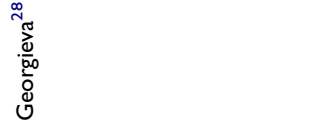 & 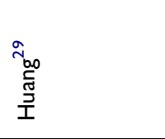 & 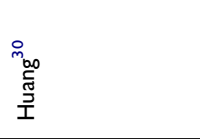 \\
\hline
\end{tabular}




\begin{tabular}{|c|c|c|c|c|c|c|c|}
\hline 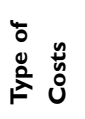 & 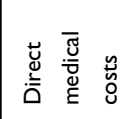 & 赵 & 赵 & 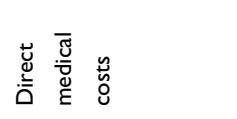 & 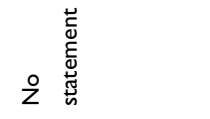 & 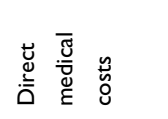 & 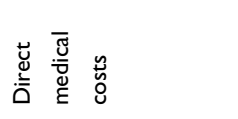 \\
\hline 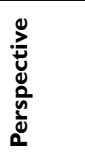 & 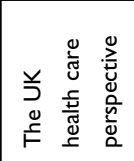 & 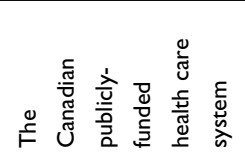 & 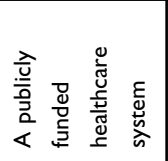 & 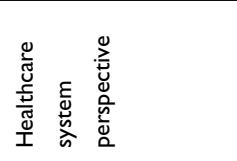 & 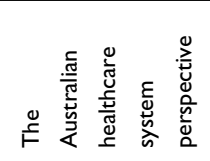 & 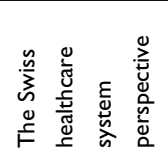 & 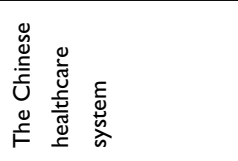 \\
\hline 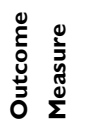 & ť̀ & 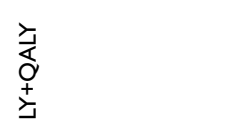 & 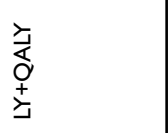 & 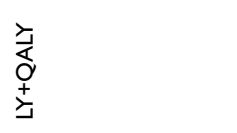 & 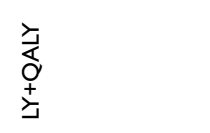 & 文 & 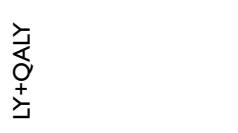 \\
\hline 䓂 & 今. & ๙े & & $\stackrel{\circ}{\dot{\sigma}}$ & ळे & & $\stackrel{\circ}{\circ}$ \\
\hline ù & 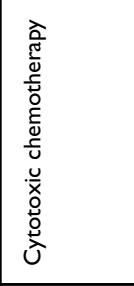 & 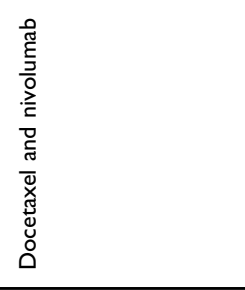 & 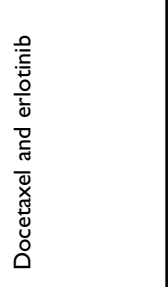 & 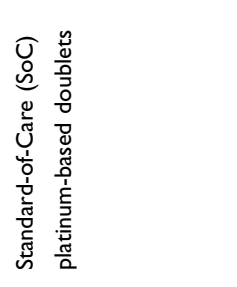 & 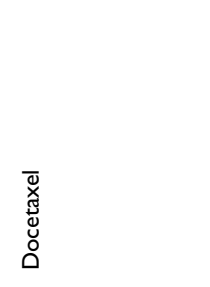 & 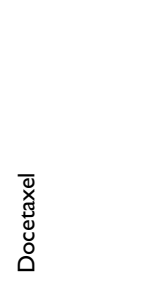 & 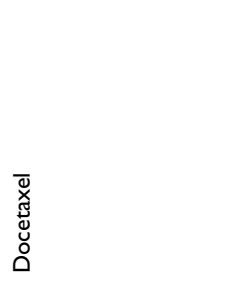 \\
\hline 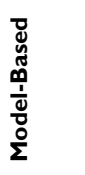 & 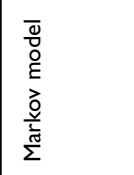 & 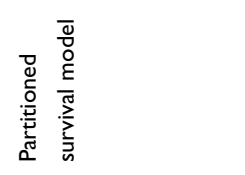 & 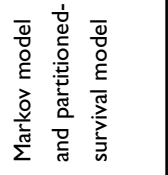 & 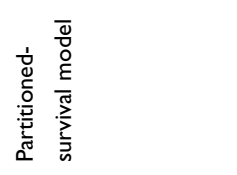 & 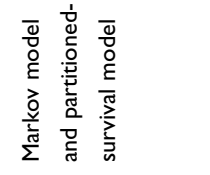 & 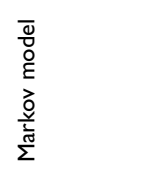 & 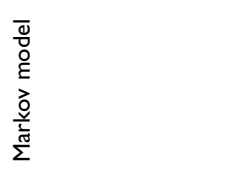 \\
\hline 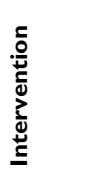 & 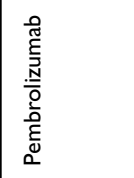 & 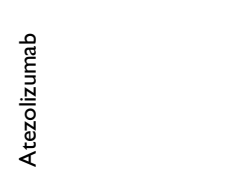 & 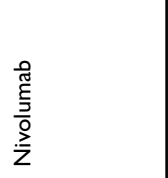 & 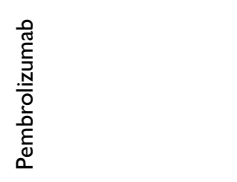 & 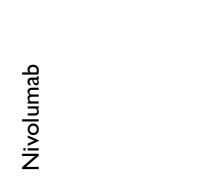 & 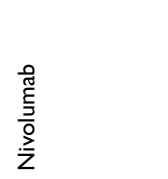 & 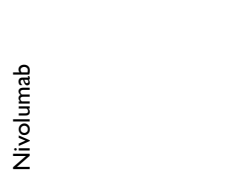 \\
\hline 疍 & 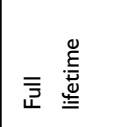 & 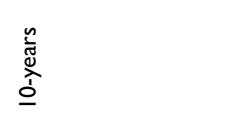 & 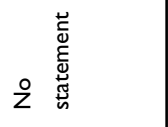 & 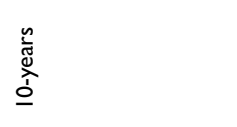 & 浐 & 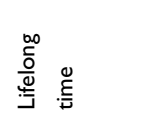 & $\overline{\overline{\bar{\Sigma}}} \stackrel{\stackrel{0}{\underline{\underline{E}}}}{\underline{\underline{\underline{E}}}}$ \\
\hline 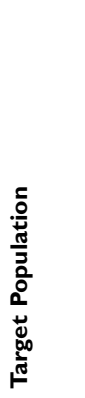 & 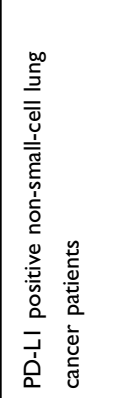 & 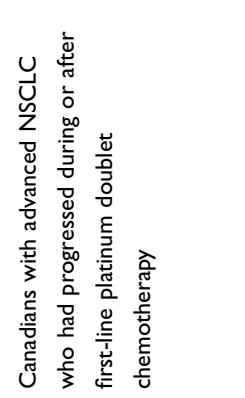 & 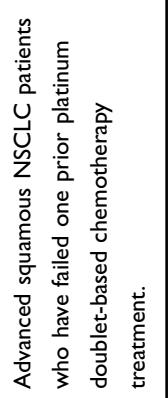 & 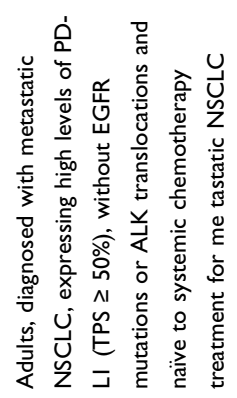 & 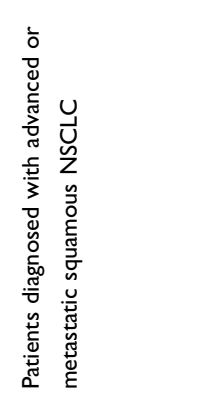 & 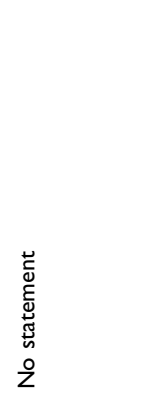 & 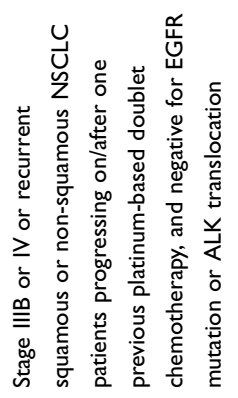 \\
\hline نे & 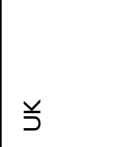 & 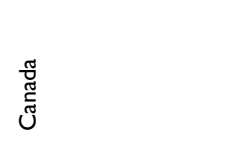 & 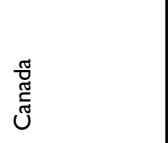 & 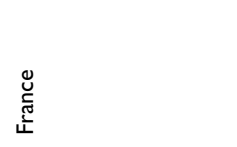 & 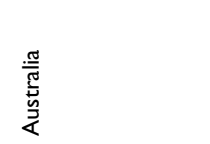 & 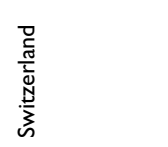 & 䄈 \\
\hline ঠ્ઇ & $\stackrel{\infty}{\frac{\infty}{2}}$ & $\stackrel{\circ}{\bar{\alpha}}$ & $\stackrel{\circ}{\circ}$ & $\frac{\circ}{\bar{\alpha}}$ & $\stackrel{\circ}{\frac{\alpha}{2}}$ & $\stackrel{\circ}{\circ}$ & $\frac{\circ}{\bar{\alpha}}$ \\
\hline 高 & $\begin{array}{l}\bar{m}_{\bar{I}} \\
\text {. }\end{array}$ & 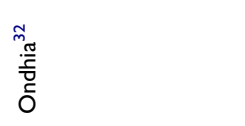 & 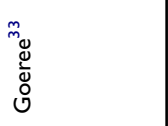 & 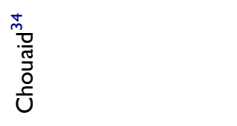 & 恕 & 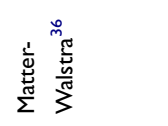 & $\bar{m}_{\Xi}$ \\
\hline
\end{tabular}




\begin{tabular}{|c|c|c|}
\hline 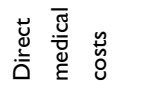 & 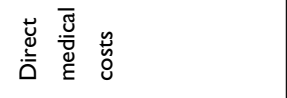 & 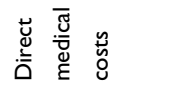 \\
\hline 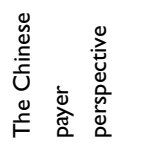 & 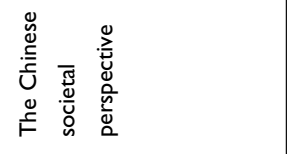 & 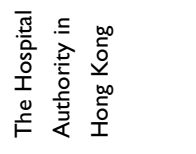 \\
\hline ¿̀ & $\frac{\grave{d}}{\partial}$ & 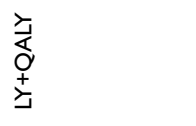 \\
\hline ஓे & ஓे & $\stackrel{\circ}{m}$ \\
\hline 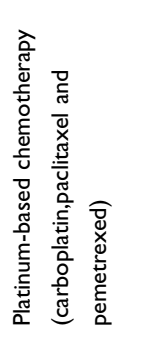 & 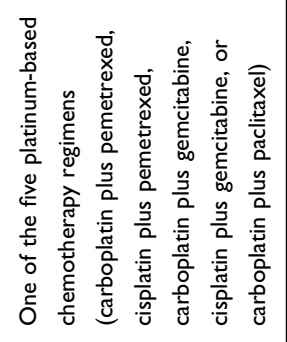 & 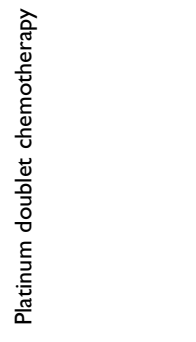 \\
\hline 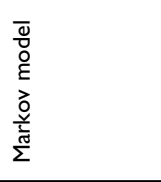 & 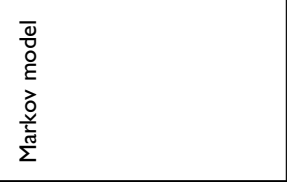 & 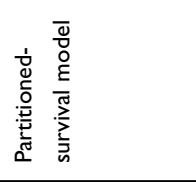 \\
\hline 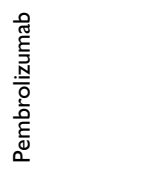 & 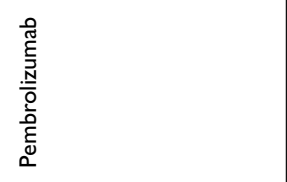 & 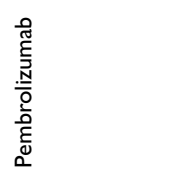 \\
\hline 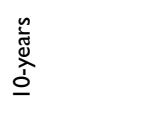 & 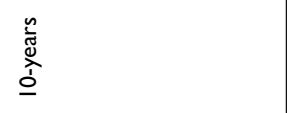 & 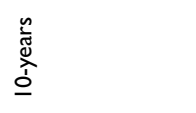 \\
\hline 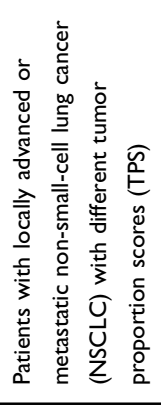 & 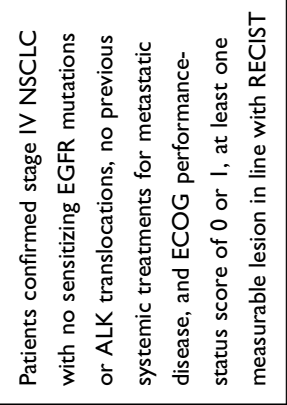 & 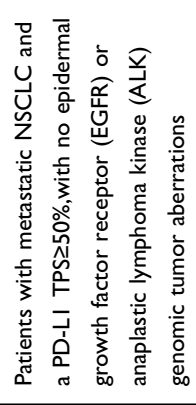 \\
\hline 苞 & 胥 & 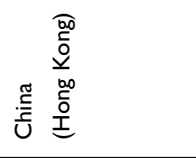 \\
\hline$\frac{\sigma}{\dot{\alpha}}$ & $\frac{a}{\dot{\alpha}}$ & $\frac{a}{i}$ \\
\hline $\begin{array}{l}\frac{\infty}{N} \\
\stackrel{0}{0} \\
\frac{D}{N}\end{array}$ & م. & 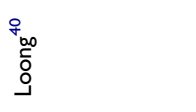 \\
\hline
\end{tabular}

\section{Quality of the Identified Studies}

We assessed the reporting quality of 21 studies by using the CHEERS checklist. The results are shown in Table 3. The scores ranged between 17 and 22.5 points, and the maximum score was 24 points. Three studies scored below 19, while two scored 17. About half of the studies ${ }^{12,22-25,28-31,33,36,38,39}$ did not provide reasons for their selected decision-analytic model nor report heterogeneity. Most studies ${ }^{12,21-28,31-34,37,39,40}$ did not mention whether there was preference measurement and evaluation. Many studies ${ }^{12,22,23,25,26,28,30,31,34,35,37,38,40}$ did not report currency, price date and conversion. And most studies did not describe how the authors were funded but only mentioned they were funded. Overall, the quality of the included studies is high (Table 3)

\section{Perspective of Analysis}

Different opinions or positions in pharmacoeconomic evaluation lead to different results. The studies included in this review indicated the following perspectives: healthcare system perspective $(\mathrm{n}=9)$, third-party healthcare payer $(n=9)$, publicly funded healthcare system $(n=2)$, and social perspective $(\mathrm{n}=1)$. With different perspectives, the research design, analysis methods, and costeffectiveness of pharmacoeconomic evaluations differed among various perspectives. Therefore, choosing a research perspective is essential.

\section{Types of Modeling Approaches and Health States}

All the studies included used models. The most commonly used models are Markov and partitioned survival (PS) models. One study used a model which was established by combining a decision tree and the Markov approach and one another study used a microsimulation model. Most Markov and PS model-based studies used the same three key health statuses: progression free, progressed disease, and death. The structure of the PS model is similar to that of the Markov model. However, unlike in a Markov model, in which transition probabilities between any two health statuses are needed, PS models directly use the data on the proportions of patients in each health status at each time point. ${ }^{30}$ Two studies ${ }^{33,35}$ compared the results of using the Markov and PS models because the authors assumed that alternative model structures or modeling approaches are typically not addressed in most economic evaluations. Many papers described good modeling practices, but only a few described how to choose from the many types of 
Table 2 Cost-Effectiveness Results

\begin{tabular}{|c|c|c|c|c|c|c|}
\hline Author & Year & Country & Perspective & Main Outcome & Threshold & ICER \\
\hline Huang $^{21}$ & 2019 & U.S. & $\begin{array}{l}\text { US third-party } \\
\text { public } \\
\text { healthcare } \\
\text { payers }\end{array}$ & $\begin{array}{l}\text { Pembrolizumab is projected to be cost- } \\
\text { effective compared with platinum-based } \\
\text { chemotherapy as first-line treatment for } \\
\text { advanced non-small-cell lung cancer with } \\
\text { PD-LI tumor proportion score } \geq 1 \% \text {. }\end{array}$ & $\$ 194,000 / \mathrm{QALY}$ & $\begin{array}{l}\text { Pembrolizumab vs platinum-based } \\
\text { chemotherapy } \$ 130,155 / \text { QALY and } \\
\$ 106,617 / L Y \text { aPD-LI } \geq 50 \% \text { patients } \\
\$ 111,781 / \text { QALY,\$9I,063/LY PD-LI } \\
\text { I-49\% patients } \$ 161,546 / \text { QALY, } \\
\$ 134,227 / L Y\end{array}$ \\
\hline She $e^{22}$ & 2019 & U.S. & $\begin{array}{l}\text { The United } \\
\text { States (US) } \\
\text { payer } \\
\text { perspective }\end{array}$ & $\begin{array}{l}\text { Pembrolizumab is a cost-effective } \\
\text { strategy compared with platinum-based } \\
\text { chemotherapy as first-line treatment in } \\
\text { locally advanced or metastatic NSCLC } \\
\text { patients with PD-LI TPS } \geq 50 \% \text { and } \\
\text { without epidermal growth factor } \\
\text { receptor (EGFR) and anaplastic } \\
\text { lymphoma kinase (ALK) mutations, but } \\
\text { not in the TPS } \geq 20 \% \text { and I\% } \\
\text { populations. }\end{array}$ & $\$ 150,000 / Q A L Y$ & $\begin{array}{l}\text { Pembrolizumab vs platinum-based } \\
\text { chemotherapy PD-LI } \geq 50 \% \text { patients } \\
\$ 136,228.82 / Q A L Y, \$ 76,390.48 / L Y \text { PD- } \\
\text { LI } \geq 20 \% \text { patients } \$ 160,625.98 / \text { QALY, } \\
\$ 90,965.81 / L Y \text { PD-LI } \geq 1 \% \text { patients } \\
\$ 179,530.17 / \text { QALY, } \$ 101,763.74 / L Y\end{array}$ \\
\hline $\mathrm{Wan}^{23}$ & 2019 & U.S. & $\begin{array}{l}\text { The United } \\
\text { States (US) } \\
\text { payer } \\
\text { perspective }\end{array}$ & $\begin{array}{l}A B C P \text { is estimated to not be cost- } \\
\text { effective compared with } B C P \text { or } C P \text { in } \\
\text { the first-line setting for patients with } \\
\text { metastatic, nonsquamous NSCLC. }\end{array}$ & $\$ 100,000 / Q A L Y$ & $\begin{array}{l}\text { ABCP vs BCP } \$ 568,967 / \text { QALY, } \\
\$ 510,865 / L Y \text { ABCP vs CP } \$ 516,114 / \\
\text { QALY, } \$ 398,657 / L Y\end{array}$ \\
\hline Weng $^{24}$ & 2019 & U.S. & $\begin{array}{l}\text { The US health } \\
\text { care system } \\
\text { perspective }\end{array}$ & $\begin{array}{l}\text { Pembrolizumab is estimated to be cost- } \\
\text { effective compared to chemotherapy for } \\
\text { previously untreated NSCLC patients } \\
\text { with different expression levels of PD- } \\
\text { LI. }\end{array}$ & $\$ 180,000 / \mathrm{QALY}$ & $\begin{array}{l}\text { Pembrolizumab vs platinum-based } \\
\text { chemotherapy PD-LI } \geq 50 \% \text { patients } \\
\$ 47,596 / \text { QALY PD-LI } \geq 20 \% \text { patients } \\
\$ 47,184 / \text { QALY PD-LI } \geq 1 \% \text { patients } \\
\$ 68,061 / \text { QALY }\end{array}$ \\
\hline Zeng $^{25}$ & 2019 & U.S. & $\begin{array}{l}\text { The US payer } \\
\text { perspective }\end{array}$ & $\begin{array}{l}\text { Pembrolizumab in addition to } \\
\text { chemotherapy provides modest } \\
\text { incremental benefit at high incremental } \\
\text { cost per QALY for the treatment of } \\
\text { previously untreated metastatic NSCLC. }\end{array}$ & $\$ 150,000 / Q A L Y$ & $\begin{array}{l}\text { Pembrolizumab plus chemotherapy vs } \\
\text { placebo plus chemotherapy } \$ 194,372 \text { / } \\
\text { QALY }\end{array}$ \\
\hline Insinga ${ }^{26}$ & 2019 & U.S. & $\begin{array}{l}\text { Third-party } \\
\text { healthcare } \\
\text { payer }\end{array}$ & $\begin{array}{l}\text { Within all relevant PD-LI sub-groups, } \\
\text { use of pembrolizumab plus } \\
\text { chemotherapy can be a cost-effective } \\
\text { first-line treatment for eligible metastatic } \\
\text { squamous NSCLC patients for whom } \\
\text { chemotherapy is currently administered. } \\
\text { In the PD-LI } \geq 50 \% \text { subgroup, additional } \\
\text { follow-up within trials of pembrolizumab } \\
\text { plus chemotherapy and pembrolizumab } \\
\text { monotherapy are needed to better } \\
\text { define cost-effectiveness between these } \\
\text { comparators. }\end{array}$ & $\$ 100,000 /$ QALY & $\begin{array}{l}\text { Pembrolizumab plus chemotherapy vs } \\
\text { chemotherapy } \$ 86,293 / \mathrm{Q} A L Y \\
\text { PD-LI } \geq 50 \% \text { patients } \$ 99,777 / \mathrm{Q} A L Y \text {, } \\
\text { PD-LI I-49\% patients } \$ 85,986 / \mathrm{QALY} \text {, } \\
\text { PD-LI<I\% patients } \$ 87,507 / \mathrm{Q} A \mathrm{LY} \text {. }\end{array}$ \\
\hline Criss $^{12}$ & 2019 & U.S. & $\begin{array}{l}\text { The US health } \\
\text { care sector }\end{array}$ & $\begin{array}{l}\text { Atezolizumab combination was not cost- } \\
\text { effective compared with bevacizumab, } \\
\text { carboplatin, and paclitaxel and provided } \\
\text { suboptimal incremental benefit } \\
\text { compared with cost vs pembrolizumab } \\
\text { combination for first-line treatment. }\end{array}$ & $\$ 100,000 / Q A L Y$ & $\begin{array}{l}\text { ABCP vs BCP } \$ 201,676 / Q A L Y \\
\text { BCP vs carboplatin plus pemetrexed } \\
\$ 80,671 / Q A L Y \text { pembrolizumab } \\
\text { combination dominated } A B C P \\
\text { pembrolizumab combination vs BCP } \\
\$ 116,698 / Q A L Y \text { pembrolizumab } \\
\text { combination vs carboplatin plus } \\
\text { pemetrexed } \$ 106,792 / Q A L Y\end{array}$ \\
\hline
\end{tabular}

(Continued) 
Table 2 (Continued).

\begin{tabular}{|c|c|c|c|c|c|c|}
\hline Author & Year & Country & Perspective & Main Outcome & Threshold & ICER \\
\hline $\operatorname{lnsinga}^{27}$ & 2018 & U.S. & $\begin{array}{l}\text { A US third- } \\
\text { party } \\
\text { healthcare } \\
\text { payer }\end{array}$ & $\begin{array}{l}\text { Although ICERs vary by subgroup and } \\
\text { comparator, results suggest } \\
\text { pembrolizumab + chemotherapy yields } \\
\text { ICERs near, and in most cases, may be } \\
\text { a cost-effective first-line treatment for } \\
\text { metastatic non-squamous NSCLC } \\
\text { patients. }\end{array}$ & $\$ 540,000 / Q A L Y$ & $\begin{array}{l}\text { Pembrolizumab+chemotherapy vs } \\
\text { chemotherapy } \$ 104,823 / \text { QALY and } \\
\$ 87,242 / L Y \text { PD-LI } \geq 50 \% \text { patients } \\
\$ 103,402 / Q A L Y, \text { PD-LI I-49\% patients } \\
\$ 66,837 / \text { QALY, PD-LI< } 1 \% \text { patients } \\
\$ 183,529 / \text { QLY. pembrolizumab } \\
\text { +chemotherapy vs pembrolizumab PD- } \\
\text { LI } \geq 50 \% \text { patients } \$ 147,51 \text { I } / \text { QALY. }\end{array}$ \\
\hline Georgieva $^{28}$ & 2018 & US and UK & $\begin{array}{l}\text { British National } \\
\text { Health System } \\
\text { (NHS) } \\
\text { perspective, US } \\
\text { cost } \\
\text { perspective }\end{array}$ & $\begin{array}{l}\text { Evidence suggests first-line } \\
\text { pembrolizumab for NSCLC may be cost- } \\
\text { effective in the US but not the UK, in } \\
\text { spite of very similar ICER values in both } \\
\text { countries. }\end{array}$ & $\begin{array}{l}\text { The UK } \$ 42,000 / \\
\text { QALY the US } \\
\$ 100,000 / \text { QALY }\end{array}$ & $\begin{array}{l}\text { Pembrolizumab vs platinum-doublets the } \\
\text { UK } \$ 52,000 \text { ( } \$ 43,000-69,000) / Q A L Y \text { the } \\
\text { US } \$ 49,000 \text { ( } \$ 40,000-67,000) / \text { QALY }\end{array}$ \\
\hline Huang $^{29}$ & 2017 & U.S. & $\begin{array}{l}\text { US third-party } \\
\text { public } \\
\text { healthcare }\end{array}$ & $\begin{array}{l}\text { Pembrolizumab is projected to be a cost- } \\
\text { effective option compared with SoC } \\
\text { platinum-based chemotherapy as first- } \\
\text { line treatment in adults with meta static } \\
\text { NSCLC expressing high levels of PD-LI. }\end{array}$ & No statement & $\begin{array}{l}\text { Pembrolizumab vs SoC \$US9762 I/QALY, } \\
\text { \$US78344/LY }\end{array}$ \\
\hline Huang $^{30}$ & 2016 & U.S. & $\begin{array}{l}\text { US third-party } \\
\text { payer } \\
\text { healthcare }\end{array}$ & $\begin{array}{l}\text { Pembrolizumab improves survival, } \\
\text { increases QALYs, and can be considered } \\
\text { as a cost-effective option compared to } \\
\text { docetaxel in PD-LI positive (TPS } \geq 50 \% \text { ) } \\
\text { pre-treated advanced NSCLC patients in } \\
\text { the US. }\end{array}$ & $\begin{array}{l}\text { No statement } \\
\text { (3-times GDP } \\
\text { per capita) }\end{array}$ & $\begin{array}{l}\text { Pembrolizumab versus docetaxel } \\
\$ 168,619 / \mathrm{QALY}\end{array}$ \\
\hline $\mathrm{Hu}^{31}$ & 2018 & UK & $\begin{array}{l}\text { The UK health } \\
\text { care } \\
\text { perspective }\end{array}$ & $\begin{array}{l}\text { Pembrolizumab is not cost-effective at its } \\
\text { current list price and a discount of } 50 \% \\
\text { or more is required for it to be cost- } \\
\text { effective comparing to commonly } \\
\text { prescribed chemotherapy. }\end{array}$ & $€ 50,000 / Q A L Y$ & $\begin{array}{l}\text { Pembrolizumab vs chemotherapy } \\
€ 86,913 / \mathrm{Q} A L Y\end{array}$ \\
\hline Ondhia $^{32}$ & 2019 & Canada & $\begin{array}{l}\text { The Canadian } \\
\text { publicly-funded } \\
\text { health care } \\
\text { system }\end{array}$ & $\begin{array}{l}\text { Atezolizumab represents a cost-effective } \\
\text { therapeutic option for the treatment of } \\
\text { patients with advanced NSCLC who } \\
\text { progress after first-line platinum doublet } \\
\text { chemotherapy. }\end{array}$ & No statement & $\begin{array}{l}\text { Atezolizumab vs docetaxel } \$ 142,074 \text { / } \\
\text { QALY, } \$ 103,726 / \text { LY atezolizumab } \\
\text { dominates nivolumab }\end{array}$ \\
\hline Goeree $^{33}$ & 2016 & Canada & $\begin{array}{l}\text { A publicly } \\
\text { funded } \\
\text { healthcare } \\
\text { system }\end{array}$ & $\begin{array}{l}\text { Nivolumab was found to involve a trade- } \\
\text { off between improved patient survival } \\
\text { and QALYs, and increased cost. It was } \\
\text { found that the use of a PS or Markov } \\
\text { model produced very similar estimates } \\
\text { of expected cost, outcomes, and } \\
\text { incremental cost-utility. }\end{array}$ & No statement & $\begin{array}{l}\text { From the PS model: nivolumab vs } \\
\text { docetaxel } \$ 151,560 / Q A L Y \text { nivolumab vs } \\
\text { erlotinib } \$ 140,601 / \text { Q } A L Y \\
\text { from the markov model: } \\
\text { nivolumab vs docetaxel } \$ 152,229 / \mathrm{Q} A L Y \\
\text { nivolumab vs erlotinib } \$ 141,838 / \mathrm{Q} A \mathrm{LY}\end{array}$ \\
\hline Chouaid $^{34}$ & 2019 & France & $\begin{array}{l}\text { Healthcare } \\
\text { system } \\
\text { perspective }\end{array}$ & $\begin{array}{l}\text { Pembrolizumab appears cost-effective } \\
\text { versus SoC chemotherapy for first-line } \\
\text { treatment of PD-LI-positive (50\%) } \\
\text { metastatic NSCLC patients. }\end{array}$ & No statement & $\begin{array}{l}\text { Squamous NSCLC: pembrolizumab vs } \\
\text { SoC } € 84,097 / Q A L Y, € 66,825 / L Y \\
\text { non-squamous NSCLC: pembrolizumab } \\
\text { vs platinum-based chemotherapies with } \\
\text { paclitaxel plus bevacizumab } € 78,729 / \\
\text { QALY,€62,846/LY }\end{array}$ \\
\hline
\end{tabular}

(Continued) 
Table 2 (Continued).

\begin{tabular}{|c|c|c|c|c|c|c|}
\hline Author & Year & Country & Perspective & Main Outcome & Threshold & ICER \\
\hline $\mathrm{Gao}^{35}$ & 2019 & Australia & $\begin{array}{l}\text { The Australian } \\
\text { healthcare } \\
\text { system } \\
\text { perspective }\end{array}$ & $\begin{array}{l}\text { The treatment with nivolumab cannot be } \\
\text { considered cost-effective. It might be } \\
\text { funded publicly by special arrangements } \\
\text { given unmet clinical needs for patients. }\end{array}$ & A $\$ 50,000 / Q A L Y$ & $\begin{array}{l}\text { Nivolumab vs docetaxel from the PS } \\
\text { model, } A \$ 198,862 / \mathrm{Q} A L Y, A \$ 181,623 / \mathrm{LY} \\
\text { from the markov model, A } \$ 220,029 / \\
\text { QALY,A } \$ 193,459 / \mathrm{LY}\end{array}$ \\
\hline $\begin{array}{l}\text { Matter- } \\
\text { Walstra }^{36}\end{array}$ & 2016 & Switzerland & $\begin{array}{l}\text { The Swiss } \\
\text { healthcare } \\
\text { system } \\
\text { perspective }\end{array}$ & $\begin{array}{l}\text { NIV compared to DOC is not cost- } \\
\text { effective for the treatment of non- } \\
\text { squamous NSCLC at current prices. } \\
\text { Price reduction, or PD-LI testing and } \\
\text { selection of patients for NIV based on } \\
\text { test positivity, improves cost- } \\
\text { effectiveness compared to DOC. }\end{array}$ & $\begin{array}{l}{ }^{\mathrm{b}} \mathrm{CHFI} 100000 / \\
\text { QALY }\end{array}$ & $\begin{array}{l}\text { Nivolumab versus docetaxel } \\
\text { CHFI77478/QALY } \\
\text { in PD-LI positive patients CHFI2489I/ } \\
\text { QALY }\end{array}$ \\
\hline $\mathrm{Liu}^{37}$ & 2019 & China & $\begin{array}{l}\text { The Chinese } \\
\text { healthcare } \\
\text { system }\end{array}$ & $\begin{array}{l}\text { Nivolumab was unable to be cost- } \\
\text { effective versus the standard second-line } \\
\text { docetaxel for patients with previously } \\
\text { treated advanced NSCLC. When } \\
\text { nivolumab is included in the NRDL, and } \\
\text { the price of nivolumab discount exceeds } \\
31.6 \% \text {, nivolumab therapy will be cost- } \\
\text { effective in China. }\end{array}$ & $\$ 28,899 / \mathrm{QALY}$ & $\begin{array}{l}\text { Nivolumab vs docetaxel } \$ 93,307 / \text { QALY } \\
\$ 74,126 / \text { LY }\end{array}$ \\
\hline Zhou $^{38}$ & 2019 & China & $\begin{array}{l}\text { The Chinese } \\
\text { payer } \\
\text { perspective }\end{array}$ & $\begin{array}{l}\text { Pembrolizumab is not a cost-effective } \\
\text { choice compared with standard } \\
\text { chemotherapy for patients with locally } \\
\text { advanced or metastatic NSCLC, } \\
\text { regardless of TPS. Deeper discount of its } \\
\text { current price would make } \\
\text { pembrolizumab a preferable choice. }\end{array}$ & $\$ 26,508 / \mathrm{QALY}$ & $\begin{array}{l}\text { Pembrolizumab vs platinum-based } \\
\text { chemotherapy } \\
\text { PD-LI } \geq 50 \% \text { patients } \$ 36,493 / \mathrm{QALY} \\
\text { PD-LI } \geq 20 \% \text { patients } \$ 42,31 \mathrm{I} / \mathrm{QALY} \\
\text { PD-LI } \geq 1 \% \text { patients } \$ 39,404 / \mathrm{QALY}\end{array}$ \\
\hline $\operatorname{Liao}^{39}$ & 2019 & China & $\begin{array}{l}\text { Chinese } \\
\text { societal } \\
\text { perspective }\end{array}$ & $\begin{array}{l}\text { Pembrolizumab is not likely to be cost- } \\
\text { effective in the treatment of PD-LI } \\
\text { positive, NSCLC for Chinese patients. } \\
\text { Less aggressive pricing may increase } \\
\text { accessibility for patients in China. }\end{array}$ & $\begin{array}{l}\text { No statement } \\
\text { (three times the } \\
\text { Chinese Gross } \\
\text { Domestic } \\
\text { Product per } \\
\text { capita) }\end{array}$ & $\$ 103,128 / \mathrm{QALY}$ \\
\hline Loong $^{40}$ & 2019 & $\begin{array}{l}\text { China } \\
\text { (Hong Kong) }\end{array}$ & $\begin{array}{l}\text { The Hospital } \\
\text { Authority in } \\
\text { Hong Kong }\end{array}$ & $\begin{array}{l}\text { Pembrolizumab in a BTS to identify } \\
\text { patients with NSCLC with PD-LI } \\
\text { TPS } \geq 50 \% \text { can be considered cost- } \\
\text { effective as first-line treatment in } \\
\text { Hong Kong compared with platinum } \\
\text { doublet chemotherapy. }\end{array}$ & $\$ 130,490 / \mathrm{QALY}$ & $\begin{array}{l}\text { Pembrolizumab vs platinum doublet } \\
\text { chemotherapy } \$ \text { I I0,922/QALY,\$89,4191 } \\
\text { LY }\end{array}$ \\
\hline
\end{tabular}

Notes: ${ }^{\text {aPD}} \mathrm{LI} \geq 50 \%$ means that PD-LI expression level is greater than or equal to $50 \%$. ${ }^{\mathrm{b}} \mathrm{CHF}$ means Swiss Franc.

Abbreviations: A, atezolizumab; B, bevacizumab; BTS, biomarker (PD-LI) test-and-treat strategy; C, carboplatin; DOC, docetaxel; GDP, gross domestic product; LY, lifeyears; P, paclitaxel; PS model, partitioned-survival model; QALY, quality-adjusted life-years; SoC, standard of chemotherapy; TPS, tumor proportion score.

available models. Markov and PS models are the two most common modelling approaches used to estimate long-term costs and effects in cancer research. ${ }^{33}$

Ten studies ${ }^{21,26,27,29,30,32-35,40}$ used the PS model. All of them used the parametric models for PFS and OS. The Weibull distributions was most used in PFS. For OS, the lognormal, the log logistic distributions were most used. All models extended the time horizon beyond the observed data and incorporated an exploration of the impact of choice of particular parametric model. The best-fitting parametric distributions was selected using statistical tests based on the Akaike information criterion and the Bayesian information criterion, combined with visual inspection.

Four studies ${ }^{26,27,30,40}$ mentioned a cohort simulation model, which is equivalent to a Markov model and is 
Table 3 CHEERS Score Results

\begin{tabular}{|c|c|c|c|c|c|}
\hline Study & Year & Yes & Ns & No & Total Scores \\
\hline Huang $^{21}$ & 2019 & 21 & 3 & 0 & 22.5 \\
\hline She ${ }^{22}$ & 2019 & 18 & 6 & 0 & 21 \\
\hline $\mathrm{Wan}^{23}$ & 2019 & 20 & 4 & 0 & 22 \\
\hline Weng $^{24}$ & 2019 & 20 & 4 & 0 & 22 \\
\hline Zeng $^{25}$ & 2019 & 16 & 8 & 0 & 20 \\
\hline Insinga ${ }^{26}$ & 2019 & 19 & 4 & I & 21 \\
\hline Criss $^{12}$ & 2019 & 17 & 6 & I & 20 \\
\hline Insinga ${ }^{27}$ & 2018 & 19 & 5 & 0 & 21.5 \\
\hline Georgieva $^{28}$ & 2018 & 17 & 5 & 2 & 19.5 \\
\hline Huang ${ }^{29}$ & 2017 & 16 & 7 & I & 19.5 \\
\hline Huang $^{30}$ & 2016 & 13 & 10 & I & 18 \\
\hline $\mathrm{Hu}^{3 \mathrm{I}}$ & 2018 & 13 & 8 & 3 & 17 \\
\hline Ondhia ${ }^{32}$ & 2019 & 18 & 6 & 0 & 21 \\
\hline Goeree $^{33}$ & 2016 & 18 & 4 & 2 & 20 \\
\hline Chouaid $^{34}$ & 2019 & 19 & 5 & 0 & 21.5 \\
\hline $\mathrm{Gao}^{35}$ & 2019 & 17 & 5 & 2 & 19.5 \\
\hline Matter-Walstra ${ }^{36}$ & 2016 & 15 & 4 & 5 & 17 \\
\hline $\operatorname{Liu}^{37}$ & 2019 & 17 & 7 & 0 & 20.5 \\
\hline Zhou ${ }^{38}$ & 2019 & 19 & 4 & I & 21 \\
\hline $\operatorname{Liao}^{39}$ & 2019 & 16 & 8 & 0 & 20 \\
\hline Loong $^{40}$ & 2019 & 18 & 6 & 0 & 21 \\
\hline
\end{tabular}

Notes: "Yes" indicates sufficiently/correctly described in the study; "No" indicates incorrect or no information regarding the specific topic in the study; "Ns" indicates not sufficiently described in the study.

used as a state-transition model. A cohort simulation model comprises the main health statuses of the disease and response to therapy. These four studies used this model to estimate health outcomes and costs for each regimen in specified patient population. The PS model divides the overall survival time into progression-free survival (PFS) and post-progression survival.

One study ${ }^{22}$ used a decision tree combined with the Markov model. However, it did not describe why this model was used. A part of the Markov model contains three statuses that are similar to those in the other included studies. Another study ${ }^{12}$ used a microsimulation model to estimate the health and cost outcomes of patients. All patients entered the model in the PFS health status and could transition to progressive disease and death, which is similar to the Markov model. Neither studies provided any model framework diagram.

\section{Uncertainty Analysis}

All studies had uncertainty analysis (sensitivity analysis). This analysis aims to explore the robustness of a model's outcomes when inputs change. Sensitivity analyses was used for model uncertainty, including multi-way probabilistic sensitivity analysis (PSA), one-way deterministic sensitivity analysis (DSA), and tornado diagram in 19, ${ }^{12,21-31,33-37,39,40}$ $19,^{12,21-31,33-40}$ and $18^{12,21-27,29-31,33-37,39,40}$ studies, respectively. Only one article ${ }^{32}$ used a scenario analysis to analyze uncertainty and did not use PSA or DSA. Another article ${ }^{28}$ used PSA but not one-way DSA and did not present a tornado diagram. One study ${ }^{38}$ only used DSA and did not present a tornado diagram. One-way DSAs were conducted to evaluate the sensitivity of the model to plausible changes in each key model parameter. Among the 19 studies that used DSA, six ${ }^{21,26,27,29,30,40}$ had tornado diagrams and showed that the parameters with the greatest effect on ICER were the extrapolations of PFS or OS RR, extrapolation of ToT for pembrolizumab, and health utility value for patients $\geq 360$ days from death. Eight other studies ${ }^{22-25,31,33,36,38}$ also reported that utility values had the largest effect on the ICER results. The price of immune checkpoint inhibitors and body weight also had a high influence. The change in some parameters, ie, median OS in pembrolizumab ${ }^{31}$ and progressive disease, ${ }^{36}$ also had a considerable influence on the results of some cases. Two-way sensitivity analyses were conducted in one study. ${ }^{39}$ This analysis varied the utility of non-progression and pembrolizumab price per milligram. The result demonstrated that the increased use of PFS coupled with reductions in pembrolizumab cost resulted in cost-effective strategies.

PSA is conducted to test the robustness of the model with respect to uncertainty in model input parameters. Each study that used PSA had different thresholds and reported different conclusions. In one study, ${ }^{28}$ the UK and US reached different conclusions. Out of 10 studies in the US, eight were about pembrolizumab and reached the same conclusion. The range of willingness-to-pay (WTP) threshold was \$50,000-200,000/QALY. The economic probability of pembrolizumab slightly differed among studies under the same threshold. When WTP $<$ $\$ 50,000 / \mathrm{QALY}$, the probability of pembrolizumab to be economical is only $15 \%$. If the WTP $<\$ 150,000 / \mathrm{QALY}$, then the economical probability is $85 \%-95 \% .^{26,27,29}$ In China, judging from the current price of pembrolizumab, it was impossible to be economical. If WTP rises to 100,000/QALY, there was 50\% economical possibility. ${ }^{39}$ Nivolumab $^{37}$ was also not cost-effective in China based on the WTP threshold value in the main regions but was $4.6 \%$ cost-effective in affluent regions. Almost all cases achieved cost-effectiveness when the WTP threshold reached $>\$ 320,000$. Nivolumab might be economical if 
WTP $>\$ 210,000 /$ QALY and \$158,000/QALY in Australia $^{35}$ and Canada, ${ }^{33}$ respectively.

\section{Health Outcome Measure}

In many countries, the preferred outcome measure for economic evaluations is QALY, which is a combined information on quality of life and duration. The CHEERS statement proposes an overview of techniques for describing health-related quality of life. Utility was applied to measure the patient's living preference during a particular health status.

In our included studies, several models derived the utilities from the published literature and clinical trials (via EQ-5D-3L instrument). The health utility values in one study ${ }^{28}$ were obtained in accordance with distributions that were consistent with a UK-based study on health utilities for advanced NSCLC treated with immunotherapy or chemotherapy. One study ${ }^{22}$ calculated health utilities on the basis of quality-of-life data (QLQ-C30 GHS/QOL) presented in the clinical trial.

\section{Findings of the Studies}

Although all studies adopted pharmacoeconomic evaluations of immunosuppressants, their inclusion criteria for populations and study of disease status differed; hence, summarizing and integrating information are slightly difficult. A total of $14,{ }^{21,22,24-31,34,38-40} 4,{ }^{33,35-37}$ and $3^{12,23,32}$ studies focused on pembrolizumab, nivolumab, and atezolizumab, respectively. In the studies on pembrolizumab, $13^{21,22,24-29,31,34,38-40}$ articles focused on the first-line treatment of NSCLC. This drug received the most attention among the immunosuppressants. Some studies compared pembrolizumab with traditional chemotherapy, while some combined it with traditional chemotherapy and then compared their individual effects. All these studies showed that pembrolizumab was highly likely to be cost-effective. However, two Chinese studies ${ }^{38,39}$ indicated that pembrolizumab was not cost-effective under current Chinese pricing. Among the included studies, nine mentioned that pembrolizumab monotherapy should be used in patients who are PD-L1 positive, and the expression of PD-L1 should be $\geq 50 \% .{ }^{9}$ Four of these eight studies $^{29,30,34,40}$ concluded that pembrolizumab was costeffective for first-line treatment compared with traditional chemotherapy. By contrast, two studies ${ }^{31,39}$ concluded that under the current threshold conditions of the country, pembrolizumab was not cost-effective. Two more articles $^{26,27}$ considered that pembrolizumab combined with chemotherapy was more economical. One study ${ }^{28}$ concluded that pembrolizumab monotherapy was economical in the US but not in the UK. However, the KEYNOTE 042 trial expanded the use of pembrolizumab to PD-L1 $\geq$ $1 \%$. Four studies ${ }^{21,22,24,38}$ were based on this trial. One study $^{38}$ indicated that pembrolizumab was not costeffective in China regardless of the expression level of PDL1. Weng ${ }^{24}$ and Huang ${ }^{21}$ concluded that pembrolizumab was cost-effective in the US regardless of the expression level of PD-L1. However, in one study, ${ }^{22}$ pembrolizumab was a cost-effective strategy compared with platinumbased chemotherapy in patients with PD-L1 TPS $\geq 50 \%$ but not $\geq 1 \%$. Insinga $2019^{26}$ compared the effects of traditional chemotherapy, pembrolizumab, and the combination of both. The author found that pembrolizumab combined with chemotherapy was more cost-effective than chemotherapy alone. The comparison between the effect of this combination to that of pembrolizumab was divided into three subgroups: PD-L1 $\geq 50 \%$, PD-L1 of $1 \%-49 \%$, and PD-L1 $<1 \%$. Substantive survival gains on pembrolizumab monotherapy were not observed in the PD-L1 $\geq 50 \%$ group compared to those on chemotherapy regimen. For patients with PD-L1 of $1-49 \%$, adding pembrolizumab to carboplatin + paclitaxel/nab-paclitaxel chemotherapy yielded a net increase of 0.98 discounted LYs compared to pembrolizumab monotherapy. For patients with PD-L1 $<1 \%$, life expectancy was projected to be doubled versus chemotherapy. Of the 14 pembrolizumab studies, 13 investigated pembrolizumab as first-line treatment. Of the 13 studies, eight showed that pembrolizumab was more economical than traditional chemotherapy regimens. One study ${ }^{28}$ included the UK and US perspectives. Pembrolizumab was economical in the US but not in the UK. Four other studies showed that using pembrolizumab was not economical given the country's current threshold. One study ${ }^{30}$ from the 14 was for included patients who experienced disease progression after undergoing platinum-containing systemic therapy. The results suggested that pembrolizumab improved quality-adjusted expectancy and could be considered a cost-effective option compared with docetaxel for patients with biomarker-identified advanced NSCLC.

Nivolumab was used for second-line treatment in the included studies. Atezolizumab could be used as firstor second-line treatment therapy. Treatment with nivolumab and atezolizumab did not explicitly require high levels of PD-L1 expression. Four studies on nivolumab did not clearly demonstrate this drug to be cost-effective. 
Goeree's research ${ }^{33}$ showed that a trade-off between improving patient survival and QALYs and increasing costs exist regardless of the cost-effectiveness of nivolumab. In addition, the cost of nivolumab is expected to increase for per patient but also improve LY and QALY outcomes compared with existing SOCs (ie, docetaxel and erlotinib). Clinical data of nivolumab in previously treated patients with squamous NSCLC presented a compelling case, that is, nivolumab represents a major advancement in disease management, where few effective treatment options are available and current treatment options are poorly tolerated with moderate efficacy. Three other nivolumab studies showed that the drug was not cost-effective at the current national threshold but could be improved by PD-L1 testing or special funding methods. Ondhia ${ }^{32}$ concluded that atezolizumab is a cost-effective second-line therapeutic option in Canada for the treatment of patients with advanced NSCLC. However, atezolizumab combination therapy was not cost-effective when used as first-line treatment for NSCLC. $\mathrm{Wan}^{23}$ and Criss ${ }^{12}$ concluded that the combination of atezolizumab, bevacizumab, carboplatin, and paclitaxel (ABCP) is less cost-effective than that of bevacizumab, carboplatin, and paclitaxel (BCP) or carboplatin and paclitaxel (CP) in the first-line treatment of patients with NSCLC. In addition, Criss ${ }^{12}$ found that atezolizumab combination provides a suboptimal increased cost benefit compared with pembrolizumab combination for first-line treatment.

\section{Discussion}

This comprehensive review examined studies on the costeffectiveness of immunotherapy for NSCLC. Twenty-one studies were included in our systematic review, and the quality of the studies was assessed using the CHEERS checklists, a well-known and trusted tool. We did not make clear restrictions with regard to the eligibility of the patients. The inclusion criteria were patients with advanced, metastatic, squamous, or non-squamous NSCLC. These studies assessed the comparison of immune checkpoint inhibitors alone with chemotherapy, or comparing immune checkpoint inhibitors in combination with traditional therapies and traditional therapies alone or immune checkpoint inhibitors alone. The models used in these studies were mainly Markov and PS models and included three statuses, namely, progression free, progressive disease, and death, to simulate the disease course. The patients started receiving treatment regimens under progression-free status. QALY was used as an outcome in all of the analyses. In most situations, a treatment is considered cost-effective when the ICER is below the threshold value.

CEAs were country specific because they included the costs of therapies applicable to each specified country. For each country, the conclusions were not completely consistent because of different clinical practice patterns, healthcare systems, cultural and ethical practices, ${ }^{21}$ and thresholds. Georgieva ${ }^{28}$ found that first-line pembrolizumab is likely to be cost-effective within the US but not in the UK compared with platinum doublets. This difference stemmed from different WTP thresholds (US: \$100,000; UK: \$42,048) as the ICER values of both countries were close to each other in nearly all sensitivity and dependency analyses. In high-income countries, such as the UK, the cost-effectiveness of pembrolizumab must be carefully considered. The contradiction between clinical efficacy and cost is important and must be resolved immediately. Countries need to find ways to lower drug prices for the benefit of more people.

The quality of the reviewed articles was generally good. The quality evaluation scores of most studies were more than 20. However, some studies scored low in measurement and valuation of preference-based outcomes, currency, price date and conversion, choice of model, heterogeneity characteristics, and source of funding. "Source-of-funding" description was among the items most frequently missed. Most studies described their source funding as from a manufacturer but did not describe how the study was funded and the role of the funder. These domain scores further showed that emphasis was given to the design of the studies and interpretation of results. This finding must be further explored to enhance the adherence to methodological standards for economic analysis or to create standards specifically for NSCLC research. Systematic, transparent, and regular assessments of publications and scrutiny of peer-review processes in dominant publication outlets are needed to improve the reporting standards of economic evaluation.

In typical pharmacoeconomic evaluations, almost all analyses were fully or partially funded by the manufacturer. In addition, most analyses clearly indicated the source of funding, and 9 studies were funded by companies. Of them, $6^{21,26,27,29,30,40}$ were funded by Merck Sharp \& Dohme Corp, the other were funded by Hoffmann-La Roche Limited, ${ }^{32}$ Bristol-Myers Squibb ${ }^{33}$ and MSD $^{34}$ respectively. The results of pharmacoeconomic research supported by the manufacturer are likely to generate 
preferences. The data cited would be biased toward the results beneficial to the pharmaceutical companies, and the results may be biased to the side beneficial to them. To improve the efficiency of data obtained for pharmacoeconomic evaluation, we should have the concepts of big data, systematize and standardize data records, strengthen the training of medical personnel about medical records, and provide objective knowledge of drug reactions.

Our review has some limitations. We did not include studies related to durvalumab because no pharmacoeconomic studies on durvalumab met our inclusion criteria. The criterion limiting the articles to those written in English meant that we might have excluded many nonEnglish studies. In addition, selecting only three databases and excluding unpublished studies (gray literature, conference abstracts) created some bias. Thus, the results may be subjected to a slight time lag. Given the small number of long-term economic evaluations, heterogeneity of the study population, differences in interventions and follow up, and huge differences between medical costs and health systems in different countries, the results must be cautiously generalized and utilized for policy-making. These factors are inherent drawbacks in economic evaluation studies. Moreover, further research must be conducted under local conditions. ${ }^{41}$

In the NCCN guideline version 3.2019 for NSCLC, single-agent pembrolizumab is the preferred drug regardless of histology for patients with metastatic NSCLC, PDL1 levels of $50 \%$ or more, and negative or unknown test results for epidermal growth factor receptor (EGFR) mutations and anaplastic lymphoma kinase (ALK) rearrangements. In our review, pembrolizumab was cost-effective in many developed countries except in the UK. For patients with metastatic non-squamous NSCLC whose PD-L1 levels are less than $50 \%$ or unknown, pembrolizumab/ carboplatin (or cisplatin)/pemetrexed is the preferred option. One study ${ }^{27}$ showed that pembrolizumab combination therapy is projected to extend life expectancy and may be a cost-effective first-line treatment for patients with metastatic non-squamous NSCLC. However, another study $^{25}$ showed that the combination of pembrolizumab and chemotherapy for patients with metastatic NSCLC had high incremental cost and modest incremental benefit. Although not an optimal biomarker, PD-L1 expression is currently the best available biomarker to assess whether patients are candidates for treatment with PD-1 or PD-L1 inhibitors. Regardless of PD-L1 expression levels, firstline combination therapies with pembrolizumab (or atezolizumab/bevacizumab)/chemotherapy are the most recommended options for patients with non-squamous NSCLC with negative test results for EGFR mutations and ALK rearrangements. However, atezolizumab combination therapy was found to be not cost-effective in two studies $^{12,23}$ conducted in the US. Pembrolizumab combination represented a better trade-off than atezolizumab combination. ${ }^{12}$ Therefore, the benefits and costs must be weighed before choosing atezolizumab combination therapy. Immune checkpoint inhibitors could not be used in patients with NSCLC and EGFR mutation and ALK translocation.

\section{Conclusions}

This review indicated that pembrolizumab regimens are costeffective in patients with NSCLC as first-line treatment in developed countries, especially in the USA. Nivolumab and atezolizumab are likely cost-effective as second-line treatment but not cost-effective as first-line treatment. However, in developing countries, such as China, less aggressive pricing may increase drug accessibility for patients with NSCLC. However, existing economic evaluations are limited by variations in the study design, outcome measure, and population characteristics. Additional research should be performed and include evidence of consistent measurements.

\section{Acknowledgment}

This study was supported by National Natural Science Foundation of China (grant number. 71804025); Science and Technology Department of Fujian Province (grant number. 2019R0054); Joint Funds for the innovation of science and Technology, Fujian province (Grant number. 2018Y9037); Fujian Provincial Health Commission Medical Innovation Project (Grant number. 2019-CX-17). The funders have no role in the study design, data collection and analysis, decision to publish, or preparation of the manuscript.

\section{Disclosure}

The authors declare that they have no conflicts of interest.

\section{References}

1. Osmani L, Askin F, Gabrielson E, Li QK. Current WHO guidelines and the critical role of immunohistochemical markers in the subclassification of non-small cell lung carcinoma (NSCLC): moving from targeted therapy to immunotherapy. Semin Cancer Biol. 2018;52(Pt 1):103-109. doi:10.1016/j.semcancer.2017.11.019

2. The L. Lung cancer: some progress, but still a lot more to do. Lancet. 2019;394:10212. 
3. Molina JR, Yang P, Cassivi SD, Schild SE, Adjei AA. Non-small cell lung cancer: epidemiology, risk factors, treatment, and survivorship. Mayo Clin Proc. 2008;83(5):584-594. doi:10.1016/S0025-6196(11)60735-0

4. Camidge DR, Pao W, Sequist LV. Acquired resistance to TKIs in solid tumours: learning from lung cancer. Nat Rev Clin Oncol. 2014;11(8):473-481. doi:10.1038/nrclinonc.2014.104

5. Herbst RS, Baas P, Kim D-W, et al. Pembrolizumab versus docetaxel for previously treated, PD-L1-positive, advanced non-small-cell lung cancer (KEYNOTE-010): a randomised controlled trial. Lancet. 2016;387(10027):1540-1550. doi:10.1016/S0140-6736(15)01281-7

6. Cho JH. Immunotherapy for non-small-cell lung cancer: current status and future obstacles. Immune Netw. 2017;17(6):378-391. doi:10.4110/in.2017.17.6.378

7. Pardoll DM. The blockade of immune checkpoints in cancer immunotherapy. Nat Rev Cancer. 2012;12(4):252-264. doi:10.1038/ nrc3239

8. Stenehjem DD, Tran D, Nkrumah MA, Gupta S. PD1/PDL1 inhibitors for the treatment of advanced urothelial bladder cancer. Onco Targets Ther. 2018;11:5973-5989. doi:10.2147/OTT.S135157

9. US Department of Health and Human Services. Pembrolizumab (KEYTRUDA) checkpoint inhibitor. Available from: http://www. fda.gov/drugs/resources-information-approved-drugs/pembrolizu mab-keytruda-checkpoint-inhibitor. Accessed November 24, 2017.

10. Mok TSK, Wu Y-L, Kudaba I, et al. Pembrolizumab versus chemotherapy for previously untreated, PD-L1-expressing, locally advanced or metastatic non-small-cell lung cancer (KEYNOTE-042): a randomised, open-label, controlled, Phase 3 trial. Lancet. 2019;393(10183):1819-1830. doi:10.1016/S01406736(18)32409-7

11. Husereau D, Drummond M, Petrou S, et al. Consolidated health economic evaluation reporting standards (CHEERS) statement. Int J Technol Assess Health Care. 2013;29(2):117-122. doi:10.1017/S0266462313000160

12. Criss SD, Mooradian MJ, Watson TR, Gainor JF, Reynolds KL, Kong CY. Cost-effectiveness of atezolizumab combination therapy for first-line treatment of metastatic nonsquamous non-small cell lung cancer in the United States. JAMA Netw Open. 2019;2(9):e1911952. doi:10.1001/jamanetworkopen.2019.11952

13. Reck M, Rodriguez-Abreu D, Robinson AG, et al. Pembrolizumab versus chemotherapy for PD-L1-positive non-small-cell lung cancer. $N$ Engl J Med. 2016;375(19):1823-1833. doi:10.1056/NEJM oa1606774

14. Gandhi L, Rodriguez-Abreu D, Gadgeel S, et al. Pembrolizumab plus chemotherapy in metastatic non-small-cell lung cancer. $N$ Engl J Med. 2018;378(22):2078-2092. doi:10.1056/NEJMoa1801005

15. Paz-Ares L, Luft A, Vicente D, et al. Pembrolizumab plus chemotherapy for squamous non-small-cell lung cancer. $N$ Engl J Med. 2018;379(21):2040-2051. doi:10.1056/NEJMoa1810865

16. Borghaei H, Paz-Ares L, Horn L, et al. Nivolumab versus docetaxel in advanced nonsquamous non-small-cell lung cancer. $N$ Engl J Med. 2015;373(17):1627-1639. doi:10.1056/NEJMoa1507643

17. Brahmer J, Reckamp KL, Baas P, et al. Nivolumab versus docetaxel in advanced squamous-cell non-small-cell lung cancer. $N$ Engl J Med. 2015;373(2):123-135. doi:10.1056/NEJMoa1504627

18. Wu YL, Lu S, Cheng Y, et al. Nivolumab versus docetaxel in a predominantly Chinese patient population with previously treated advanced NSCLC: checkMate 078 randomized Phase iii clinical trial. J Thorac Oncol. 2019;14(5):867-875. doi:10.1016/j.jtho.2019.01.006

19. Gadgeel SM, Lukas RV, Goldschmidt J, et al. Atezolizumab in patients with advanced non-small cell lung cancer and history of asymptomatic, treated brain metastases: exploratory analyses of the phase III OAK study. Lung Cancer. 2019;128:105-112. doi:10.1016/ j.lungcan.2018.12.017

20. Socinski MA, Jotte RM, Cappuzzo F, et al. Atezolizumab for first-line treatment of metastatic nonsquamous NSCLC. $N$ Engl J Med. 2018;378(24):2288-2301. doi:10.1056/NEJMoa1716948
21. Huang M, Lopes GL, Insinga RP, et al. Cost-effectiveness of pembrolizumab versus chemotherapy as first-line treatment in PD-L1positive advanced non-small-cell lung cancer in the USA. Immunotherapy. 2019;11(17):1463-1478. doi:10.2217/imt-20190178

22. She L, Hu H, Liao M, et al. Cost-effectiveness analysis of pembrolizumab versus chemotherapy as first-line treatment in locally advanced or metastatic non-small cell lung cancer with PD-L1 tumor proportion score $1 \%$ or greater. Lung Cancer. 2019;138:88-94. doi:10.1016/j.lungcan.2019.10.017

23. Wan X, Luo X, Tan C, Zeng X, Zhang Y, Peng L. First-line atezolizumab in addition to bevacizumab plus chemotherapy for metastatic, nonsquamous non-small cell lung cancer: a United States-based cost-effectiveness analysis. Cancer. 2019;125 (20):3526-3534. doi:10.1002/cncr.32368

24. Weng X, Luo S, Lin S, et al. Cost-utility analysis of pembrolizumab versus chemotherapy as first-line treatment for metastatic non-small cell lung cancer with different PD-L1 expression levels. Oncol Res. 2019.

25. Zeng X, Wan X, Peng L, et al. Cost-effectiveness analysis of pembrolizumab plus chemotherapy for previously untreated metastatic non-small cell lung cancer in the USA. BMJ Open. 2019;9(12): e031019. doi:10.1136/bmjopen-2019-031019

26. Insinga RP, Vanness DJ, Feliciano JL, et al. Cost-effectiveness of pembrolizumab in combination with chemotherapy versus chemotherapy and pembrolizumab monotherapy in the first-line treatment of squamous non-small-cell lung cancer in the US. Curr Med Res Opin. 2019;35(7):1241-1256. doi:10.1080/ 03007995.2019.1571297

27. Insinga RP, Vanness DJ, Feliciano JL, Vandormael K, Traore S, Burke T. Cost-effectiveness of pembrolizumab in combination with chemotherapy in the 1st line treatment of non-squamous NSCLC in the US. J Med Econ. 2018;21(12):1191-1205. doi:10.1080/ 13696998.2018.1521416

28. Georgieva M, da Silveira Nogueira Lima JP, Aguiar P Jr, de Lima Lopes G Jr, Haaland B. Cost-effectiveness of pembrolizumab as first-line therapy for advanced non-small cell lung cancer. Lung Cancer. 2018;124:248-254. doi:10.1016/j.lungcan.2018.08.018

29. Huang M, Lou Y, Pellissier J, et al. Cost effectiveness of pembrolizumab vs. standard-of-care chemotherapy as first-line treatment for metastatic NSCLC that expresses high levels of PD-L1 in the United States. Pharmacoeconomics. 2017;35(8):831-844. doi:10.1007/ s40273-017-0527-z

30. Huang M, Lou Y, Pellissier J, et al. Cost-effectiveness of pembrolizumab versus docetaxel for the treatment of previously treated PD-L1 positive advanced NSCLC patients in the United States. J Med Econ. 2017;20(2):140-150. doi:10.1080/13696998.2016.1230123

31. Hu X, Hay JW. First-line pembrolizumab in PD-L1 positive non-small-cell lung cancer: A cost-effectiveness analysis from the UK health care perspective. Lung Cancer. 2018;123:166-171. doi:10.1016/j.lungcan.2018.07.012

32. Ondhia U, Conter HJ, Owen S, et al. Cost-effectiveness of second-line atezolizumab in Canada for advanced non-small cell lung cancer (NSCLC). J Med Econ. 2019;22(7):625-637. doi:10.1080/13696998.2019.1590842

33. Goeree R, Villeneuve J, Goeree J, Penrod JR, Orsini L, Tahami Monfared AA. Economic evaluation of nivolumab for the treatment of second-line advanced squamous NSCLC in Canada: a comparison of modeling approaches to estimate and extrapolate survival outcomes. J Med Econ. 2016;19(6):630-644. doi:10.3111/ 13696998.2016.1151432

34. Chouaid C, Bensimon L, Clay E, et al. Cost-effectiveness analysis of pembrolizumab versus standard-of-care chemotherapy for first-line treatment of PD-L1 positive ( $>50 \%)$ metastatic squamous and nonsquamous non-small cell lung cancer in France. Lung Cancer. 2019;127:44-52. doi:10.1016/j.lungcan.2018.11.008 
35. Gao L, Li SC. Modelled economic evaluation of nivolumab for the treatment of second-line advanced or metastatic squamous non-smallcell lung cancer in Australia using both partition survival and Markov models. Appl Health Econ Health Policy. 2019;17(3):371-380. doi:10.1007/s40258-018-0452-0

36. Matter-Walstra K, Schwenkglenks $M$, Aebi $S$, et al. A cost-effectiveness analysis of nivolumab versus docetaxel for advanced nonsquamous NSCLC including PD-L1 testing. $J$ Thorac Oncol. 2016;11(11):1846-1855. doi:10.1016/j.jtho.2016.05.032

37. Liu Q, Luo X, Peng L, et al. Nivolumab versus docetaxel for previously treated advanced non-small cell lung cancer in China: a cost-effectiveness analysis. Clin Drug Investig. 2019.

38. Zhou K, Jiang C, Li Q. Cost-effectiveness analysis of pembrolizumab monotherapy and chemotherapy in the non-small-cell lung cancer with different PD-L1 tumor proportion scores. Lung Cancer. 2019;136:98-101. doi:10.1016/j.lungcan.2019.08.028
39. Liao W, Huang J, Hutton D, Li Q. Cost-effectiveness analysis of first-line pembrolizumab treatment for PD-L1 positive, non-small cell lung cancer in China. J Med Econ. 2019;22(4):344-349. doi:10.1080/ 13696998.2019.1570221

40. Loong $\mathrm{HH}$, Wong CKH, Leung LKS, et al. Cost effectiveness of PD-L1-based test-and-treat strategy with pembrolizumab as the first-line treatment for metastatic NSCLC in Hong Kong. Pharmacoecon Open. 2019.

41. Littlewood C, Ashton J, Chance-Larsen K, May S, Sturrock B. The quality of reporting might not reflect the quality of the study: implications for undertaking and appraising a systematic review. J Man Manip Ther. 2012;20(3):130-134. doi:10.1179/2042618611Y.0000000013

\section{Publish your work in this journal}

Cancer Management and Research is an international, peer-reviewed open access journal focusing on cancer research and the optimal use of preventative and integrated treatment interventions to achieve improved outcomes, enhanced survival and quality of life for the cancer patient.
The manuscript management system is completely online and includes a very quick and fair peer-review system, which is all easy to use. Visit http://www.dovepress.com/testimonials.php to read real quotes from published authors. 